\title{
C-N Difference Schemes for Dissipative Symmetric Regularized Long Wave Equations with Damping Term
}

\author{
Jinsong $\mathrm{Hu}^{1}{ }^{1}$ Bing $\mathrm{Hu}{ }^{2}$ and Youcai $\mathrm{Xu}^{2}$ \\ ${ }^{1}$ School of Mathematics and Computer Engineering, Xihua University, Chengdu 610039, China \\ ${ }^{2}$ School of Mathematics, Sichuan University, Chengdu 610064, China
}

Correspondence should be addressed to Bing Hu, hbscu@yahoo.com.cn

Received 25 November 2010; Revised 16 February 2011; Accepted 25 February 2011

Academic Editor: Francesco Pellicano

Copyright (c) 2011 Jinsong Hu et al. This is an open access article distributed under the Creative Commons Attribution License, which permits unrestricted use, distribution, and reproduction in any medium, provided the original work is properly cited.

We study the initial-boundary problem of dissipative symmetric regularized long wave equations with damping term. Crank-Nicolson nonlinear-implicit finite difference scheme is designed. Existence and uniqueness of numerical solutions are derived. It is proved that the finite difference scheme is of second-order convergence and unconditionally stable by the discrete energy method. Numerical simulations verify the theoretical analysis.

\section{Introduction}

A symmetric version of regularized long wave equation (SRLWE),

$$
\begin{gathered}
u_{x x t}-u_{t}=\rho_{x}+u u_{x}, \\
\rho_{t}+u_{x}=0
\end{gathered}
$$

has been proposed to model the propagation of weakly nonlinear ion acoustic and space charge waves [1]. The sech ${ }^{2}$ solitary wave solutions are

$$
\begin{aligned}
& u(x, t)=\frac{3\left(v^{2}-1\right)}{v} \operatorname{sech}^{2} \frac{1}{2} \sqrt{\frac{v^{2}-1}{v^{2}}}(x-v t), \\
& \rho(x, t)=\frac{3\left(v^{2}-1\right)}{v^{2}} \operatorname{sech}^{2} \frac{1}{2} \sqrt{\frac{v^{2}-1}{v^{2}}}(x-v t) .
\end{aligned}
$$


The four invariants and some numerical results have been obtained in [1], where $v$ is the velocity, $v^{2}>1$. Obviously, eliminating $\rho$ from (1.1), we get a class of SRLWE

$$
u_{t t}-u_{x x}+\frac{1}{2}\left(u^{2}\right)_{x t}-u_{x x t t}=0
$$

Equation (1.3) is explicitly symmetric in the $x$ and $t$ derivatives and is very similar to the regularized long wave equation that describes shallow water waves and plasma drift waves $[2,3]$. The SRLW equation also arises in many other areas of mathematical physics [4-6]. Numerical investigation indicates that interactions of solitary waves are inelastic [7] thus, the solitary wave of the SRLWE is not a solution. Research on the well-posedness for its solution and numerical methods has aroused more and more interest. In [8], Guo studied the existence, uniqueness and regularity of the numerical solutions for the periodic initial value problem of generalized SRLW by the spectral method. In [9], Zheng et al. presented a Fourier pseudospectral method with a restraint operator for the SRLWEs, and proved its stability and obtained the optimum error estimates. There are other methods such as pseudospectral method, finite difference method for the initial-boundary value problem of SRLWEs (see [10-15]).

Because of gravity and resistance of propagation medium and air, the principle of dissipation must be considered when studying the move of nonlinear wave. In applications, the viscous damping effect is inevitable and it plays the same important role as the dispersive effect. Therefore, it is more significant to study the dissipative symmetric regularized long wave equations with the damping term

$$
\begin{gathered}
u_{x x t}-u_{t}+v u_{x x}=\rho_{x}+u u_{x}, \\
\rho_{t}+u_{x}+\gamma \rho=0,
\end{gathered}
$$

where $v, \gamma$ are positive, $v>0$ is the dissipative coefficient, $\gamma>0$ is the damping coefficient. Equation (1.4) are a reasonable model to render essential phenomena of nonlinear ion acoustic wave motion when dissipation is considered (see [16-20]). Existence, uniqueness and well-posedness of global solutions to (1.4) are presented (see [16-20]). But it is difficult to find the analytical solution to (1.4), which makes numerical solution important.

In this paper, we study (1.4) with

$$
u(x, 0)=u_{0}(x), \quad \rho(x, 0)=\rho_{0}(x), \quad x \in\left[x_{L}, x_{R}\right]
$$

and the boundary conditions

$$
u\left(x_{L}, t\right)=u\left(x_{R}, t\right)=0, \quad \rho\left(x_{L}, t\right)=\rho\left(x_{R}, t\right)=0, \quad t \in[0, T] .
$$

In [21] we proposed a three-level implicit finite difference scheme to (1.4)-(1.6) with secondorder convergence. But the three-level implicit finite difference scheme can not start by itself, we need to select other two-level schemes (such as the C-N Scheme) to get $u^{1}, \rho^{1}$. Then, reusing initial vale $u^{0}, \rho^{0}$, we can work out $u^{2}, \rho^{2}, u^{3}, \rho^{3}, \ldots$ Since the form of SRLW equations is similar ro the Rosenau equation and Rosenau-Burgers equation, the established difference 
schemes in $[22,23]$ for solving Rosenau equation and Rosenau-Burgers equation are helpful to investigate the SRLWEs. We propose the Crank-Nicolson finite difference scheme for (1.4)(1.6) which can start by itself. We will show that this difference scheme is uniquely solvable, convergent and stable in both theoretical and numerical senses.

\section{Finite Difference Scheme and Its Error Estimation}

Let $h$ and $\tau$ be the uniform step size in the spatial and temporal direction, respectively. Denote $x_{j}=x_{L}+j h(j=0,1,2, \ldots, J), t_{n}=n \tau(n=0,1,2, \ldots, N), N=[T / \tau], u_{j}^{n} \approx u\left(x_{j}, t_{n}\right), \rho_{j}^{n} \approx$ $\rho\left(x_{j}, t_{n}\right)$ and $Z_{h}^{0}=\left\{u=\left(u_{j}\right) \mid u_{0}=u_{J}=0, j=0,1,2, \ldots, J\right\}$. Throughout this paper, we will denote $C$ as a generic constant independent of $h$ and $\tau$ that varies in the context. We define the difference operators, inner product, and norms that will be used in this paper as follows:

$$
\begin{aligned}
& \left(u_{j}^{n}\right)_{x}=\frac{u_{j+1}^{n}-u_{j}^{n}}{h}, \quad\left(u_{j}^{n}\right)_{\bar{x}}=\frac{u_{j}^{n}-u_{j-1}^{n}}{h}, \quad\left(u_{j}^{n}\right)_{\hat{x}}=\frac{u_{j+1}^{n}-u_{j-1}^{n}}{2 h}, \quad\left(u_{j}^{n}\right)_{t}=\frac{u_{j}^{n+1}-u_{j}^{n}}{\tau}, \\
& u_{j}^{n+1 / 2}=\frac{u_{j}^{n+1}+u_{j}^{n}}{2}, \quad\left\langle u^{n}, v^{n}\right\rangle=h \sum_{j=1}^{J-1} u_{j}^{n} v_{j}^{n}, \quad\left\|u^{n}\right\|^{2}=\left\langle u^{n}, u^{n}\right\rangle, \quad\left\|u^{n}\right\|_{\infty}=\max _{1 \leq j \leq J-1}\left|u_{j}^{n}\right| .
\end{aligned}
$$

Then, the Crank-Nicolson finite difference scheme for the solution of (1.4)-(1.6) is as follows:

$$
\begin{gathered}
\left(u_{j}^{n}\right)_{t}-\left(u_{j}^{n}\right)_{x \bar{x} t}+\left(\rho_{j}^{n+1 / 2}\right)_{\widehat{x}}-v\left(u_{j}^{n+1 / 2}\right)_{x \bar{x}}+\frac{1}{3}\left(u_{j+1}^{n+1 / 2}+u_{j}^{n+1 / 2}+u_{j-1}^{n+1 / 2}\right)\left(u_{j}^{n+1 / 2}\right)_{\widehat{x}}=0, \\
\left(\rho_{j}^{n}\right)_{t}+\left(u_{j}^{n+1 / 2}\right)_{\widehat{x}}+\gamma \rho_{j}^{n+1 / 2}=0, \\
u_{j}^{0}=u_{0}\left(x_{j}\right), \quad \rho_{j}^{0}=\rho_{0}\left(x_{j}\right), \quad 1 \leq j \leq J-1, \\
u_{0}^{n}=u_{J}^{n}=0, \quad \rho_{0}^{n}=\rho_{J}^{n}=0, \quad 1 \leq n \leq N .
\end{gathered}
$$

Lemma 2.1. It follows summation by parts $[12,23]$ that for any two discrete functions $u, v \in Z_{h^{\prime}}^{0}$

$$
\left\langle u_{x}, v\right\rangle=-\left\langle u, v_{\bar{x}}\right\rangle, \quad\left\langle u_{x \bar{x}}, v\right\rangle=-\left\langle u_{x}, v_{x}\right\rangle .
$$

Lemma 2.2 (Discrete Sobolev's inequality $[12,23]$ ). There exist two constants $C_{1}$ and $C_{2}$ such that

$$
\left\|u^{n}\right\|_{\infty} \leq C_{1}\left\|u^{n}\right\|+C_{2}\left\|u_{x}^{n}\right\| .
$$


Lemma 2.3 (Discrete Gronwall's inequality $[12,23])$. Suppose $w(k), \rho(k)$ are nonnegative function and $\rho(k)$ is nondecreasing. If $C>0$ and $w(k) \leq \rho(k)+C \tau \sum_{l=0}^{k-1} w(l)$, then

$$
w(k) \leq \rho(k) e^{C \tau k}
$$

Theorem 2.4. If $u_{0} \in H^{1}, \rho_{0} \in L_{2}$, then the solution of (2.2)-(2.5) satisfies

$$
\left\|u^{n}\right\| \leq C, \quad\left\|u_{x}^{n}\right\| \leq C, \quad\left\|\rho^{n}\right\| \leq C, \quad\left\|u^{n}\right\|_{\infty} \leq C, \quad(n=1,2, \ldots, N) .
$$

Proof. Taking an inner product of (2.2) with $2 u^{n+1 / 2}$ (i.e., $u^{n+1}+u^{n}$ ) and considering the boundary condition (2.5) and Lemma 2.1, we obtain

$$
\begin{gathered}
\frac{1}{\tau}\left(\left\|u^{n+1}\right\|^{2}-\left\|u^{n}\right\|^{2}\right)+\frac{1}{\tau}\left(\left\|u_{x}^{n+1}\right\|^{2}-\left\|u_{x}^{n}\right\|^{2}\right)+\left\langle\rho_{\widehat{x}}^{n+1 / 2}, 2 u^{n+1 / 2}\right\rangle \\
-v\left\langle u_{x \bar{x}}^{n+1 / 2}, 2 u^{n+1 / 2}\right\rangle+\left\langle P^{n+1 / 2}, 2 u^{n+1 / 2}\right\rangle=0,
\end{gathered}
$$

where $P_{j}^{n+1 / 2}=(1 / 3)\left(u_{j+1}^{n+1 / 2}+u_{j}^{n+1 / 2}+u_{j-1}^{n+1 / 2}\right) \cdot\left(u_{j}^{n+1 / 2}\right)_{\widehat{x}}$.

Since

$$
\begin{gathered}
\left\langle\rho_{\widehat{x}}^{n+1 / 2}, 2 u^{n+1 / 2}\right\rangle=-2\left\langle u_{\widehat{x}}^{n+1 / 2}, \rho^{n+1 / 2}\right\rangle, \\
\left\langle u_{x \bar{x}}^{n+1 / 2}, 2 u^{n+1 / 2}\right\rangle=-2\left\|u_{x}^{n+1 / 2}\right\|^{2}, \\
\left\langle P^{n+1 / 2}, 2 u^{n+1 / 2}\right\rangle=2 h \sum_{j=1}^{J-1} P_{j}^{n+1 / 2} u_{j}^{n+1 / 2} \\
=\frac{2}{3} h \sum_{j=1}^{J-1}\left(u_{j+1}^{n+1 / 2}+u_{j}^{n+1 / 2}+u_{j-1}^{n+1 / 2}\right) \cdot\left(u_{j}^{n+1 / 2}\right)_{\widehat{x}} u_{j}^{n+1 / 2} \\
=\frac{1}{3} \sum_{j=1}^{J-1}\left(u_{j+1}^{n+1 / 2}+u_{j}^{n+1 / 2}+u_{j-1}^{n+1 / 2}\right) \cdot\left(u_{j+1}^{n+1 / 2}-u_{j-1}^{n+1 / 2}\right) u_{j}^{n+1 / 2} \\
=\frac{1}{3} \sum_{j=1}^{J-1}\left(u_{j+1}^{n+1 / 2}+u_{j}^{n+1 / 2}\right) u_{j+1}^{n+1 / 2} u_{j}^{n+1 / 2} \\
-\frac{1}{3} \sum_{j=1}^{J-1}\left(u_{j}^{n+1 / 2}+u_{j-1}^{n+1 / 2}\right) u_{j-1}^{n+1 / 2} u_{j}^{n+1 / 2}=0
\end{gathered}
$$

we obtain

$$
\frac{1}{\tau}\left(\left\|u^{n+1}\right\|^{2}-\left\|u^{n}\right\|^{2}\right)+\frac{1}{\tau}\left(\left\|u_{x}^{n+1}\right\|^{2}-\left\|u_{x}^{n}\right\|^{2}\right)-2\left\langle u_{\hat{x}}^{n+1 / 2}, \rho^{n+1 / 2}\right\rangle+2 v\left\|u_{x}^{n+1 / 2}\right\|^{2}=0 .
$$


Taking an inner product of (2.3) with $2 \rho^{n+1 / 2}$ (i.e., $\rho^{n+1}+\rho^{n}$ ) and considering the boundary condition (2.5) and Lemma 2.1, we obtain

$$
\frac{1}{\tau}\left(\left\|\rho^{n+1}\right\|^{2}-\left\|\rho^{n}\right\|^{2}\right)+\left\langle u_{\widehat{x}}^{n+1 / 2}, 2 \rho^{n+1 / 2}\right\rangle+2 \gamma\left\|\rho^{n+1 / 2}\right\|^{2}=0
$$

Adding (2.13) to (2.14), we have

$$
\begin{gathered}
\left(\left\|u^{n+1}\right\|^{2}-\left\|u^{n}\right\|^{2}\right)+\left(\left\|u_{x}^{n+1}\right\|^{2}-\left\|u_{x}^{n}\right\|^{2}\right)+\left(\left\|\rho^{n+1}\right\|^{2}-\left\|\rho^{n}\right\|^{2}\right) \\
=-2 \tau\left(v\left\|u_{x}^{n+1 / 2}\right\|^{2}+r\left\|\rho^{n+1 / 2}\right\|^{2}\right) \leq 0
\end{gathered}
$$

which implies

$$
\left\|u^{n}\right\|^{2}+\left\|u_{x}^{n}\right\|^{2}+\left\|\rho^{n}\right\|^{2} \leq\left\|u^{n-1}\right\|^{2}+\left\|u_{x}^{n-1}\right\|^{2}+\left\|\rho^{n-1}\right\|^{2} \leq \cdots \leq\left\|u^{0}\right\|^{2}+\left\|u_{x}^{0}\right\|^{2}+\left\|\rho^{0}\right\|^{2}=C .
$$

Then, it holds

$$
\left\|u^{n}\right\| \leq C, \quad\left\|u_{x}^{n}\right\| \leq C, \quad\left\|\rho^{n}\right\| \leq C .
$$

By Lemma 2.2, we obtain $\left\|u^{n}\right\|_{\infty} \leq C$.

Theorem 2.5. Assume that $u^{0} \in H^{2}, \rho^{0} \in H^{1}$, the solution of difference scheme (2.2)-(2.5) satisfies:

$$
\left\|\rho_{x}^{n}\right\| \leq C, \quad\left\|u_{x x}^{n}\right\| \leq C, \quad\left\|u_{x}^{n}\right\| \leq C, \quad\left\|\rho^{n}\right\|_{\infty} \leq C, \quad(n=1,2, \ldots, N) .
$$

Proof. Differentiating backward (2.2)-(2.5) with respect to $x$, we obtain

$$
\begin{gathered}
\left(u_{j}^{n}\right)_{x t}-\left(u_{j}^{n}\right)_{x x \bar{x} t}+\left(\rho_{j}^{n+1 / 2}\right)_{x \widehat{x}}-v\left(u_{j}^{n+1 / 2}\right)_{x x \bar{x}}+\left(P_{j}^{n+1 / 2}\right)_{x}=0, \\
\left(\rho_{j}^{n}\right)_{x t}+\left(u_{j}^{n+1 / 2}\right)_{x \widehat{x}}+\gamma\left(\rho_{j}^{n+1 / 2}\right)_{x}=0, \\
\left(u_{j}^{0}\right)_{x}=u_{0, x}\left(x_{j}\right), \quad\left(\rho_{j}^{0}\right)_{x}=\rho_{0, x}\left(x_{j}\right), \quad 1 \leq j \leq J-1, \\
\left(u_{0}^{n}\right)_{x}=\left(u_{J}^{n}\right)_{x}=0, \quad\left(\rho_{0}^{n}\right)_{x}=\left(\rho_{J}^{n}\right)_{x}=0, \quad 1 \leq n \leq N,
\end{gathered}
$$

where $\left(P_{j}^{n+1 / 2}\right)_{x}=(1 / 3)\left[\left(u_{j+1}^{n+1 / 2}+u_{j}^{n+1 / 2}+u_{j-1}^{n+1 / 2}\right)\left(u_{j}^{n+1 / 2}\right)_{\hat{x}}\right]_{x}$. 
Computing the inner product of (2.19) with $2 u_{x}^{n+1 / 2}$ (i.e., $u_{x}^{n+1}+u_{x}^{n}$ ) and considering (2.22) and Lemma 2.1, we obtain

$$
\begin{aligned}
& \frac{1}{\tau}\left(\left\|u_{x}^{n+1}\right\|^{2}-\left\|u_{x}^{n}\right\|^{2}\right)+\frac{1}{\tau}\left(\left\|u_{x x}^{n+1}\right\|^{2}-\left\|u_{x x}^{n}\right\|^{2}\right) \\
& \quad+\left\langle\rho_{x \bar{x}}^{n+1 / 2}, 2 u_{x}^{n+1 / 2}\right\rangle-v\left\langle u_{x x \bar{x}}^{n+1 / 2}, 2 u_{x}^{n+1 / 2}\right\rangle+\left\langle P_{x}^{n+1 / 2}, 2 u_{x}^{n+1 / 2}\right\rangle=0 .
\end{aligned}
$$

It follows from Theorem 2.4 that

$$
\left|u_{j+1}^{n+1 / 2}+u_{j}^{n+1 / 2}+u_{j-1}^{n+1 / 2}\right| \leq C, \quad(j=0,1,2, \ldots, J) .
$$

By the Schwarz inequality and Lemma 2.1, we get

$$
\begin{aligned}
\left\langle P_{x}^{n+1 / 2}, 2 u_{x}^{n+1 / 2}\right\rangle & =-2\left\langle P^{n+1 / 2}, u_{x \bar{x}}^{n+1 / 2}\right\rangle \\
& =-\frac{2}{3} h \sum_{j=1}^{J-1}\left(u_{j+1}^{n+1 / 2}+u_{j}^{n+1 / 2}+u_{j-1}^{n+1 / 2}\right) \cdot\left(u_{j}^{n+1 / 2}\right)_{\hat{x}}\left(u_{j}^{n+1 / 2}\right)_{x \bar{x}} \\
& \leq C h \sum_{j=1}^{J-1}\left|\left(u_{j}^{n+1 / 2}\right)_{\hat{x}}\right| \cdot\left|\left(u_{j}^{n+1 / 2}\right)_{x \bar{x}}\right| \leq C\left(\left\|u_{x}^{n+1 / 2}\right\|^{2}+\left\|u_{x x}^{n+1 / 2}\right\|^{2}\right) \\
& \leq C\left(\left\|u_{x}^{n+1}\right\|^{2}+\left\|u_{x}^{n}\right\|^{2}+\left\|u_{x x}^{n+1}\right\|^{2}+\left\|u_{x x}^{n}\right\|^{2}\right) .
\end{aligned}
$$

By

$$
\begin{gathered}
\left\langle\rho_{x \hat{x}}^{n+1 / 2}, 2 u_{x}^{n+1 / 2}\right\rangle=-2\left\langle u_{x \hat{x}}^{n+1 / 2}, \rho_{x}^{n+1 / 2}\right\rangle, \\
\left\langle u_{x x \bar{x}}^{n+1 / 2}, 2 u_{x}^{n+1 / 2}\right\rangle=-2\left\|u_{x x}^{n+1 / 2}\right\|^{2},
\end{gathered}
$$

it follows from (2.23) that

$$
\begin{aligned}
& \left(\left\|u_{x}^{n+1}\right\|^{2}-\left\|u_{x}^{n}\right\|^{2}\right)+\left(\left\|u_{x x}^{n+1}\right\|^{2}-\left\|u_{x x}^{n}\right\|^{2}\right)-2 \tau\left\langle u_{x \widehat{x}}^{n+1 / 2}, \rho_{x}^{n+1 / 2}\right\rangle \\
& \leq-2 v \tau\left\|u_{x x}^{n+1 / 2}\right\|^{2}+C \tau\left(\left\|u_{x}^{n+1}\right\|^{2}+\left\|u_{x}^{n}\right\|^{2}+\left\|u_{x x}^{n+1}\right\|^{2}+\left\|u_{x x}^{n}\right\|^{2}\right) .
\end{aligned}
$$

Computing the inner product of (2.20) with $2 \rho_{x}^{n+1 / 2}$ (i.e., $\rho_{x}^{n+1}+\rho_{x}^{n}$ ) and considering (2.22) and Lemma 2.1, we obtain

$$
\left(\left\|\rho_{x}^{n+1}\right\|^{2}-\left\|\rho_{x}^{n}\right\|^{2}\right)+2 \tau\left\langle u_{x \hat{x}}^{n+1 / 2}, \rho_{x}^{n+1 / 2}\right\rangle+2 \gamma \tau\left\|\rho_{x}^{n+1 / 2}\right\|^{2}=0 .
$$


Adding (2.28) to (2.27), we have

$$
\begin{aligned}
& \left(\left\|u_{x}^{n+1}\right\|^{2}-\left\|u_{x}^{n}\right\|^{2}\right)+\left(\left\|u_{x x}^{n+1}\right\|^{2}-\left\|u_{x x}^{n}\right\|^{2}\right)+\left(\left\|\rho_{x}^{n+1}\right\|^{2}-\left\|\rho_{x}^{n}\right\|^{2}\right) \\
& \leq-2 v \tau\left\|u_{x x}^{n+1 / 2}\right\|^{2}-2 \gamma \tau\left\|\rho_{x}^{n+1 / 2}\right\|^{2}+C \tau\left(\left\|u_{x}^{n+1}\right\|^{2}+\left\|u_{x}^{n}\right\|^{2}+\left\|u_{x x}^{n+1}\right\|^{2}+\left\|u_{x x}^{n}\right\|^{2}\right) \\
& \leq C \tau\left(\left\|u_{x}^{n+1}\right\|^{2}+\left\|u_{x}^{n}\right\|^{2}+\left\|u_{x x}^{n+1}\right\|^{2}+\left\|u_{x x}^{n}\right\|^{2}+\left\|\rho_{x}^{n+1}\right\|^{2}+\left\|\rho_{x}^{n}\right\|^{2}\right) .
\end{aligned}
$$

Let $A^{n}=\left\|u_{x}^{n}\right\|^{2}+\left\|u_{x x}^{n}\right\|^{2}+\left\|\rho_{x}^{n}\right\|^{2}$, we obtain $A^{n+1}-A^{n} \leq C \tau\left(A^{n+1}+A^{n}\right)$.

Choosing suitable $\tau$ which is small enough to satisfy $1-C \tau>0$, we get

$$
A^{n+1}-A^{n} \leq C \tau A^{n}
$$

Summing up (2.30) from 0 to $n-1$, we have

$$
A^{n} \leq A^{0}+C \tau \sum_{l=0}^{n-1} A^{l}
$$

By Lemma 2.3, we get $A^{n} \leq C$, which implies $\left\|\rho_{x}^{n}\right\| \leq C$, $\left\|u_{x x}^{n}\right\| \leq C$. It follows from Theorem 2.4 and Lemma 2.2 that $\left\|u_{x}^{n}\right\|_{\infty} \leq C,\left\|\rho^{n}\right\|_{\infty} \leq C$.

\section{Solvability, Convergence, and Stability}

The following Brouwer fixed point theorem will be needed in order to show the existence of solution for (2.2)-(2.5). For the proof, see [24].

Lemma 3.1 (Brouwer fixed point theorem). Let $H$ be a finite dimensional inner product space, suppose that $g: H \rightarrow H$ is continuous and there exists an $\alpha>0$ such that $<g(x), x>0$ for all $x \in H$ with $\|x\|=\alpha$. Then there exists $x^{*} \in H$ such that $g\left(x^{*}\right)=0$ and $\left\|x^{*}\right\| \leq \alpha$.

Let $Z_{\Delta}=\left\{v=\left(v_{1}, v_{2}\right)=\left(v_{1, j}, v_{2, j}\right) \mid v_{1,0}=v_{1, J}=v_{2,0}=v_{2, J}=0, j=0,1,2, \ldots, J\right\}$, equipped with the inner product $\left\langle v, v^{\prime}\right\rangle=\left\langle\left(v_{1}, v_{2}\right),\left(v_{1}^{\prime}, v_{2}^{\prime}\right)\right\rangle=\left\langle v_{1}, v_{1}^{\prime}\right\rangle+\left\langle v_{2}, v_{2}^{\prime}\right\rangle$ and the norm $\|v\|^{2}=\left\|v_{1}\right\|^{2}+\left\|v_{2}\right\|^{2}$.

Theorem 3.2. There exists $\left(u^{n}, \rho^{n}\right) \in Z_{\Delta}$ which satisfies the difference scheme (2.2)-(2.5).

Proof. In order to prove the theorem by the mathematical induction, we assume that $\left(u^{0}, \rho^{0}\right)$, $\left(u^{1}, \rho^{1}\right), \ldots,\left(u^{n}, \rho^{n}\right) \in Z_{\Delta}$ satisfying $(2.2)-(2.5)$. Next prove there exists $\left(u^{n+1}, \rho^{n+1}\right)$ which satisfies (2.2)-(2.5).

Let $g=\left(g_{1}, g_{2}\right)$ be a operator on $Z_{\Delta}$ defined by

$$
\begin{gathered}
g_{1}(v)=2 v_{1}-2 u^{n}-2 v_{1 x \bar{x}}+2 u_{x \bar{x}}^{n}+\tau v_{2 \widehat{x}}-v \tau v_{1 x \bar{x}}+\tau W \\
g_{2}(v)=2 v_{2}-2 \rho^{n}+\tau v_{1 \widehat{x}}+\gamma \tau v_{2}, \quad \forall v=\left(v_{1}, v_{2}\right) \in Z_{\Delta},
\end{gathered}
$$


where $W_{j}=(1 / 3)\left(v_{1, j+1}+v_{1, j}+v_{1, j-1}\right)\left(v_{1, j}\right)_{\widehat{x}}$. Computing the inner product of (3.1) with $v=\left(v_{1}, v_{2}\right)$, similarly to (2.11) and (2.12), we obtain

$$
\left\langle v_{2 \hat{x}}, v_{1}\right\rangle=-\left\langle v_{1 \hat{x}}, v_{2}\right\rangle, \quad\left\langle W, v_{1}\right\rangle=0
$$

By (2.5) and the Schwarz inequality, we obtain

$$
\begin{aligned}
\langle g(v), v\rangle= & \left\langle g_{1}(v), v_{1}\right\rangle+\left\langle g_{2}(v), v_{2}\right\rangle \\
= & 2\left\|v_{1}\right\|^{2}-2\left\langle u^{n}, v_{1}\right\rangle+2\left\|v_{1 x}\right\|^{2}-2\left\langle u_{x}^{n}, v_{1 x}\right\rangle+v \tau\left\|v_{1 x}\right\|^{2}+2\left\|v_{2}\right\|^{2} \\
& -2\left\langle\rho^{n}, v_{2}\right\rangle+\gamma \tau\left\|v_{2}\right\|^{2} \\
\geq & 2\left\|v_{1}\right\|^{2}-\left(\left\|u^{n}\right\|^{2}+\left\|v_{1}\right\|^{2}\right)+2\left\|v_{1 x}\right\|^{2}-\left(\left\|u_{x}^{n}\right\|^{2}+\left\|v_{1 x}\right\|^{2}\right)+v \tau\left\|v_{1 x}\right\|^{2} \\
& +2\left\|v_{2}\right\|^{2}-\left(\left\|\rho^{n}\right\|^{2}+\left\|v_{2}\right\|^{2}\right)+\gamma \tau\left\|v_{2}\right\|^{2} \\
= & \left\|v_{1}\right\|^{2}+\left\|v_{2}\right\|^{2}+\left\|v_{1 x}\right\|^{2}-\left\|u^{n}\right\|^{2}-\left\|u_{x}^{n}\right\|^{2}-\left\|\rho^{n}\right\|^{2}+v \tau\left\|v_{1 x}\right\|^{2}+\gamma \tau\left\|v_{2}\right\|^{2} \\
\geq & \|v\|^{2}-\left(\left\|u^{n}\right\|^{2}+\left\|u_{x}^{n}\right\|^{2}+\left\|\rho^{n}\right\|^{2}\right) .
\end{aligned}
$$

Hence it is obvious that $\left\langle g(v), v>0\right.$ for all $v \in Z_{\Delta}$ with $\|v\|^{2}=\left(\left\|u^{n}\right\|^{2}+\left\|u_{x}^{n}\right\|^{2}+\left\|\rho^{n}\right\|^{2}\right)+1$. It follows from Lemma 3.1 that there exists $v^{*} \in Z_{\Delta}$ such that $g\left(v^{*}\right)=0$. If we take $u^{n+1}=$ $2 v_{1}-u^{n}, \rho^{n+1}=2 v_{2}^{*}-\rho^{n}$, then $\left(u^{n+1}, \rho^{n+1}\right)$ satisfies (2.2)-(2.5). This completes the proof.

Next we show that the difference scheme (2.2)-(2.5) is convergent and stable.

Let $v(x, t)$ and $\varnothing(x, t)$ be the solution of problem (1.4)-(1.6), that is, $v_{j}^{n}=u\left(x_{j}, t_{n}\right)$, $\varnothing_{j}^{n}=\rho\left(x_{j}, t_{n}\right)$, then the truncation of the difference scheme (2.2)-(2.5) is

$$
\begin{gathered}
r_{j}^{n}=\left(v_{j}^{n}\right)_{t}-\left(v_{j}^{n}\right)_{x \bar{x} t}+\left(\varnothing_{j}^{n+1 / 2}\right)_{\widehat{x}}-v\left(v_{j}^{n+1 / 2}\right)_{x \bar{x}}+\frac{1}{3}\left(v_{j+1}^{n+1 / 2}+v_{j}^{n+1 / 2}+v_{j-1}^{n+1 / 2}\right)\left(v_{j}^{n+1 / 2}\right)_{\widehat{x}^{\prime}} \\
s_{j}^{n}=\left(\varnothing_{j}^{n}\right)_{t}+\left(v_{j}^{n+1 / 2}\right)_{\widehat{x}}+\gamma \varnothing_{j}^{n+1 / 2} .
\end{gathered}
$$

Making use of Taylor expansion, we know that $\left|r_{j}^{n}\right|+\left|s_{j}^{n}\right|=O\left(\tau^{2}+h^{2}\right)$ hold if $h, \tau \rightarrow 0$.

Lemma 3.3. Suppose that $u_{0} \in H^{1}, \rho_{0} \in L_{2}$, the solution of (1.4)-(1.6) satisfies $\|u\|_{L_{2}} \leq C$, $\left\|u_{x}\right\|_{L_{2}} \leq$ $C,\|\rho\|_{L_{2}} \leq C$ and $\|u\|_{L_{\infty}} \leq C$.

Proof. See Lemma 1.1 in [21]. 
Theorem 3.4. Suppose $u_{0} \in H^{1}, \rho_{0} \in L_{2}$, then the solution $u^{n}$ and $\rho^{n}$ to the difference scheme (2.2)(2.5) converges to the solution of problem (1.4)-(1.6), and the rate of convergence is $O\left(\tau^{2}+h^{2}\right)$.

Proof. Subtracting (2.2) from (3.4) and subtracting (2.3) from (3.5), letting $e_{j}^{n}=v_{j}^{n}-u_{j}^{n}, \eta_{j}^{n}=$ $\phi_{j}^{n}-\rho_{j}^{n}$, we have

$$
\begin{gathered}
r_{j}^{n}=\left(e_{j}^{n}\right)_{t}-\left(e_{j}^{n}\right)_{x \bar{x} t}+\left(\eta_{j}^{n+1 / 2}\right)_{\widehat{x}}-v\left(e_{j}^{n+1 / 2}\right)_{x \bar{x}}+Q_{j}^{n+1 / 2}, \\
s_{j}^{n}=\left(\eta_{j}^{n}\right)_{t}+\left(e_{j}^{n+1 / 2}\right)_{\hat{x}}+\gamma \eta_{j}^{n+1 / 2},
\end{gathered}
$$

where

$$
Q_{j}^{n+1 / 2}=\frac{1}{3}\left(v_{j+1}^{n+1 / 2}+v_{j}^{n+1 / 2}+v_{j-1}^{n+1 / 2}\right) \cdot\left(v_{j}^{n+1 / 2}\right)_{\widehat{x}}-\frac{1}{3}\left(u_{j+1}^{n+1 / 2}+u_{j}^{n+1 / 2}+u_{j-1}^{n+1 / 2}\right) \cdot\left(u_{j}^{n+1 / 2}\right)_{\widehat{x}} .
$$

Computing the inner product of (3.6) with $2 e^{n+1 / 2}$ we get

$$
\begin{aligned}
\left\langle r^{n}, 2 e^{n+1 / 2}\right\rangle= & \frac{1}{\tau}\left(\left\|e^{n+1}\right\|^{2}-\left\|e^{n}\right\|^{2}\right)+\frac{1}{\tau}\left(\left\|e_{x}^{n+1}\right\|^{2}-\left\|e_{x}^{n}\right\|^{2}\right)+2 v\left\|e_{x}^{n+1 / 2}\right\|^{2} \\
& +\left\langle\eta_{\hat{x}}^{n+1 / 2}, 2 e^{n+1 / 2}\right\rangle+\left\langle Q^{n+1 / 2}, 2 e^{n+1 / 2}\right\rangle .
\end{aligned}
$$

Similarly to (2.11), we have

$$
\left\langle\eta_{\widehat{x}}^{n+1 / 2}, 2 e^{n+1 / 2}\right\rangle=-2\left\langle e_{\widehat{x}}^{n+1 / 2}, \eta^{n+1 / 2}\right\rangle
$$

Then (3.9) can be changed to

$$
\begin{array}{r}
\left(\left\|e^{n+1}\right\|^{2}-\left\|e^{n}\right\|^{2}\right)+\left(\left\|e_{x}^{n+1}\right\|^{2}-\left\|e_{x}^{n}\right\|^{2}\right)-2 \tau\left\langle e_{\hat{x}}^{n+1 / 2}, \eta^{n+1 / 2}\right\rangle \\
=-2 v \tau\left\|e_{x}^{n+1 / 2}\right\|^{2}+\tau\left\langle r^{n}, 2 e^{n+1 / 2}\right\rangle+\tau\left\langle-Q^{n+1 / 2}, 2 e^{n+1 / 2}\right\rangle .
\end{array}
$$

It follow from Lemma 3.3, Theorems 2.4 and 2.5 that

$$
\left|v_{j+1}^{n+1 / 2}+v_{j}^{n+1 / 2}+v_{j-1}^{n+1 / 2}\right| \leq C, \quad\left|\left(u_{j}^{n+1 / 2}\right)\right| \leq C, \quad(j=0,1,2, \ldots, J) .
$$


Since

$$
\begin{aligned}
\left\langle-Q^{n+1 / 2}, 2 e^{n+1 / 2}\right\rangle= & -\frac{2}{3} h \sum_{j=1}^{J-1}\left(v_{j+1}^{n+1 / 2}+v_{j}^{n+1 / 2}+v_{j-1}^{n+1 / 2}\right)\left(v_{j}^{n+1 / 2}\right)_{\hat{x}} e_{j}^{n+1 / 2} \\
& +\frac{2}{3} h \sum_{j=1}^{J-1}\left(u_{j+1}^{n+1 / 2}+u_{j}^{n+1 / 2}+u_{j-1}^{n+1 / 2}\right)\left(u_{j}^{n+1 / 2}\right)_{\hat{x}} e_{j}^{n+1 / 2} \\
= & -\frac{2}{3} h \sum_{j=1}^{J-1}\left(v_{j+1}^{n+1 / 2}+v_{j}^{n+1 / 2}+v_{j-1}^{n+1 / 2}\right)\left(e_{j}^{n+1 / 2}\right)_{\widehat{x}} e_{j}^{n+1 / 2} \\
& -\frac{2}{3} h \sum_{j=1}^{J-1}\left(e_{j+1}^{n+1 / 2}+e_{j}^{n+1 / 2}+e_{j-1}^{n+1 / 2}\right)\left(u_{j}^{n+1 / 2}\right)_{\widehat{x}} e_{j}^{n+1 / 2},
\end{aligned}
$$

and the Schwarz inequality, we obtain

$$
\begin{aligned}
\left\langle-Q^{n+1 / 2}, 2 e^{n+1 / 2}\right\rangle \leq & \frac{2}{3} C h \sum_{j=1}^{J-1}\left|\left(e_{j}^{n+1 / 2}\right)_{\hat{x}}\right| \cdot\left|e_{j}^{n+1 / 2}\right| \\
& +\frac{2}{3} C h \sum_{j=1}^{J-1}\left(\left|e_{j+1}^{n+1 / 2}\right|+\left|e_{j}^{n+1 / 2}\right|+\left|e_{j-1}^{n+1 / 2}\right|\right)\left|e_{j}^{n+1 / 2}\right| \\
\leq & C\left(\left\|e^{n+1 / 2}\right\|^{2}+\left\|e_{x}^{n+1 / 2}\right\|^{2}\right) \leq C\left(\left\|e^{n+1}\right\|^{2}+\left\|e^{n}\right\|^{2}+\left\|e_{x}^{n+1}\right\|^{2}+\left\|e_{x}^{n}\right\|^{2}\right) .
\end{aligned}
$$

According to

$$
\left\langle r^{n}, 2 e^{n+1 / 2}\right\rangle=\left\langle r^{n}, e^{n+1}+e^{n}\right\rangle \leq\left\|r^{n}\right\|^{2}+\frac{1}{2}\left[\left\|e^{n+1}\right\|^{2}+\left\|e^{n}\right\|^{2}\right] .
$$

It follows from (3.14), (3.15), and (3.11) that

$$
\begin{gathered}
\left(\left\|e^{n+1}\right\|^{2}-\left\|e^{n}\right\|^{2}\right)+\left(\left\|e_{x}^{n+1}\right\|^{2}-\left\|e_{x}^{n}\right\|^{2}\right)-2 \tau\left\langle e_{\hat{x}}^{n+1 / 2}, \eta^{n+1 / 2}\right\rangle \\
\leq C \tau\left[\left\|e^{n+1}\right\|^{2}+\left\|e^{n}\right\|^{2}+\left\|e_{x}^{n+1}\right\|^{2}+\left\|e_{x}^{n}\right\|^{2}\right]+\tau\left\|r^{n}\right\|^{2} .
\end{gathered}
$$

Computing the inner product of (3.7) with $2 \eta^{n+1 / 2}$, we obtain

$$
\begin{aligned}
\left(\left\|\eta^{n+1}\right\|^{2}-\left\|\eta^{n}\right\|^{2}\right)+2 \tau\left\langle e_{\widehat{x}}^{n+1 / 2}, \eta^{n+1 / 2}\right\rangle & =\tau\left\langle s^{n}, 2 \eta^{n+1 / 2}\right\rangle-2 \gamma \tau\left\|\eta^{n+1 / 2}\right\|^{2} \\
& \leq C \tau\left[\left\|\eta^{n+1}\right\|^{2}+\left\|\eta^{n}\right\|^{2}\right]+\tau\left\|s^{n}\right\|^{2} .
\end{aligned}
$$


Adding (3.17) to (3.16) we have

$$
\begin{aligned}
& \left(\left\|e^{n+1}\right\|^{2}-\left\|e^{n}\right\|^{2}\right)+\left(\left\|e_{x}^{n+1}\right\|^{2}-\left\|e_{x}^{n}\right\|^{2}\right)+\left\|\eta^{n+1}\right\|^{2}-\left\|\eta^{n}\right\|^{2} \\
& \quad \leq \tau\left\|r^{n}\right\|^{2}+\tau\left\|s^{n}\right\|^{2}+C \tau\left[\left\|e^{n+1}\right\|^{2}+\left\|e^{n}\right\|^{2}+\left\|e_{x}^{n+1}\right\|^{2}+\left\|e_{x}^{n}\right\|^{2}+\left\|\eta^{n+1}\right\|^{2}+\left\|\eta^{n}\right\|^{2}\right] .
\end{aligned}
$$

Let $B^{n}=\left\|e^{n}\right\|^{2}+\left\|e_{x}^{n}\right\|^{2}+\left\|\eta^{n}\right\|^{2}$, we get

$$
B^{n+1}-B^{n} \leq \tau\left\|r^{n}\right\|^{2}+\tau\left\|s^{n}\right\|^{2}+C \tau\left(B^{n+1}+B^{n}\right) .
$$

If $\tau$ is sufficiently small which satisfies $1-C \tau>0$, then

$$
B^{n+1}-B^{n} \leq C \tau B^{n}+C \tau\left\|r^{n}\right\|^{2}+C \tau\left\|s^{n}\right\|^{2} .
$$

Summing up (3.20) from 0 to $n-1$, we have

$$
B^{n} \leq B^{0}+C \tau \sum_{l=0}^{n-1}\left\|r^{l}\right\|^{2}+C \tau \sum_{l=0}^{n-1}\left\|s^{l}\right\|^{2}+C \tau \sum_{l=0}^{n-1} B^{l} .
$$

Since

$$
\begin{aligned}
& \tau \sum_{l=0}^{n-1}\left\|r^{l}\right\|^{2} \leq n \tau \max _{0 \leq l \leq n-1}\left\|r^{l}\right\|^{2} \leq T \cdot O\left(\tau^{2}+h^{2}\right)^{2}, \\
& \tau \sum_{l=0}^{n-1}\left\|s^{l}\right\|^{2} \leq n \tau \max _{0 \leq l \leq n-1}\left\|s^{l}\right\|^{2} \leq T \cdot O\left(\tau^{2}+h^{2}\right)^{2}
\end{aligned}
$$

and $B^{0}=O\left(\tau^{2}+h^{2}\right)^{2}$, we obtain

$$
B^{n} \leq O\left(\tau^{2}+h^{2}\right)^{2}+C \tau \sum_{l=0}^{n-1} B^{l}
$$

From Lemma 2.3, we get

$$
B^{n} \leq O\left(\tau^{2}+h^{2}\right)^{2}
$$

which implies

$$
\left\|e^{n}\right\| \leq O\left(\tau^{2}+h^{2}\right), \quad\left\|e_{x}^{n}\right\| \leq O\left(\tau^{2}+h^{2}\right), \quad\left\|\eta^{n}\right\| \leq O\left(\tau^{2}+h^{2}\right) .
$$


Table 1: The error comparison in the sense of $l_{\infty}$ at various time step when $v=\gamma=0.2$.

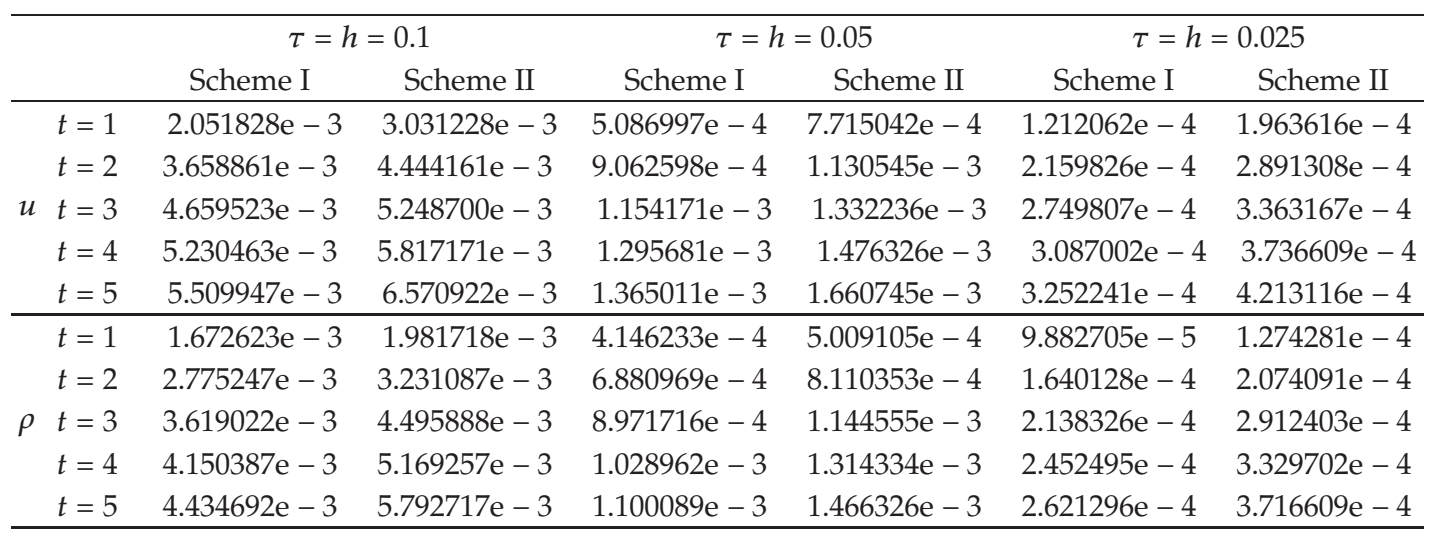

Using Lemma 2.2, we get

$$
\left\|e^{n}\right\|_{\infty} \leq O\left(\tau^{2}+h^{2}\right)
$$

Similarly to Theorem 3.4, we can prove the results as follows.

Theorem 3.5. Under the conditions of Theorem 3.4, the solution $u^{n}$ and $\rho^{n}$ of (2.2)-(2.5) is stable in the senses of norm $\|\cdot\|_{\infty}$ and $\|\cdot\|_{L^{2}}$, respectively.

Theorem 3.6. The solution $u^{n}$ of (2.2)-(2.5) is unique.

\section{Numerical Simulations}

The difference scheme (2.2)-(2.5) is a nonlinear system about $u_{j}^{n+1}$ that can be easily solved by Newton iterative algorithm. When $t=0$, the damping does not effect and the dissipative term will not appear. So the initial conditions (1.4)-(1.6) are same as those of (1.1):

$$
u_{0}(x)=\frac{5}{2} \operatorname{sech}^{2} \frac{\sqrt{5}}{6} x, \quad \rho_{0}(x)=\frac{5}{3} \operatorname{sech}^{2} \frac{\sqrt{5}}{6} x, \quad(v=1.5) .
$$

Let $x_{L}=-20, x_{R}=20, T=5.0$. Since we do not know the exact solution of (1.4), an error estimates method in [23] is used: A comparison between the numerical solutions on a coarse mesh and those on a refine mesh is made. We consider the solution on mesh $\tau=h=1 / 160$ as the reference solution. We denote the $\mathrm{C}-\mathrm{N}$ scheme in this paper as Scheme I and the difference scheme in [21] as Scheme II. In Tables 1 and 2 we give the ratios in the sense of $l_{\infty}$ at various time step when $v=\gamma=0.2$ and $v=\gamma=0.5$, respectively.

In Tables 3 and 4 we verify the second convergence of the scheme I using the method in [25] when $v=\gamma=0.2$ and $v=\gamma=0.5$, respectively.

When $v=\gamma=0.2$ and $v=\gamma=0.5$, a wave figure comparison of $u$ and $\rho$ at various time step with $\tau=h=0.05$ is as follow: (see Figures 1, 2, 3, and 4). 
Table 2: The error comparison in the sense of $l_{\infty}$ at various time step when $v=\gamma=0.5$.

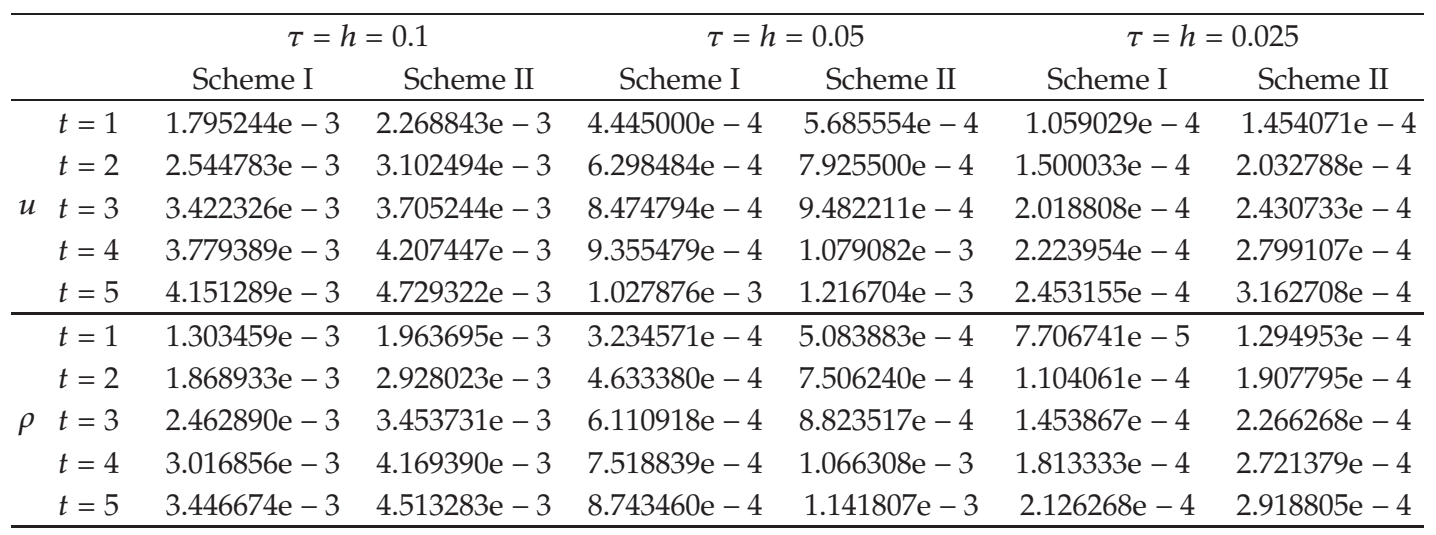

Table 3: The verification of the second convergence when $v=r=0.2$.

\begin{tabular}{lcccccc}
\hline & \multicolumn{3}{c}{$\left\|e^{n}(h, \tau)\right\|_{\infty} /\left\|e^{2 n}(h / 2, \tau / 2)\right\|_{\infty}$} & \multicolumn{3}{c}{$\left\|\eta^{n}(h, \tau)\right\|_{\infty} /\left\|\eta^{2 n}(h / 2, \tau / 2)\right\|_{\infty}$} \\
& $\tau=h=0.1$ & $\tau=h=0.05$ & $\tau=h=0.025$ & $\tau=h=0.1$ & $\tau=h=0.05$ & $\tau=h=0.025$ \\
\hline$t=1$ & - & 4.03347 & 4.19698 & - & 4.03408 & 4.19544 \\
$t=2$ & - & 4.03732 & 4.19596 & - & 4.03322 & 4.19539 \\
$t=3$ & - & 4.03711 & 4.19728 & - & 4.03381 & 4.19567 \\
$t=4$ & - & 4.03684 & 4.19721 & - & 4.03357 & 4.19557 \\
$t=5$ & - & 4.03656 & 4.19714 & - & 4.03121 & 4.19674 \\
\hline
\end{tabular}

Table 4: The verification of the second convergence when $v=\gamma=0.5$.

\begin{tabular}{ccccccc}
\hline & \multicolumn{3}{c}{$\left\|e^{n}(h, \tau)\right\|_{\infty} /\left\|e^{2 n}(h / 2, \tau / 2)\right\|_{\infty}$} & \multicolumn{3}{c}{$\left\|\eta^{n}(h, \tau)\right\|_{\infty} /\left\|\eta^{2 n}(h / 2, \tau / 2)\right\|_{\infty}$} \\
& $\tau=h=0.1$ & $\tau=h=0.05$ & $\tau=h=0.025$ & $\tau=h=0.1$ & $\tau=h=0.05$ & $\tau=h=0.025$ \\
\hline$t=1$ & - & 4.03879 & 4.19724 & - & 4.02977 & 4.19707 \\
$t=2$ & - & 4.04031 & 4.19807 & - & 4.03326 & 4.19667 \\
$t=3$ & - & 4.03824 & 4.19792 & - & 4.03031 & 4.20322 \\
$t=4$ & - & 4.03976 & 4.20669 & - & 4.01240 & 4.14642 \\
$t=5$ & - & 4.03871 & 4.19002 & - & 3.94200 & 4.11211 \\
\hline
\end{tabular}

\section{Conclusion}

In this paper, we propose Crank-Nicolson nonlinear-implicit finite difference scheme of the initial-boundary problem of dissipative symmetric regularized long wave equations with damping term. The two-levels finite difference scheme is of second-order convergence and unconditionally stable, which can start by itself. From the Tables 1 and 2 we conclude that the $\mathrm{C}-\mathrm{N}$ scheme is more efficient than the Scheme 2 in [21]. From the Tables 3 and 4 we conclude that the C-N scheme is of second-order convergence obviously. Figures 1-4 show that the height of wave crest is more and more low with time elapsing due to the effect of damping term and dissipative term and when $v, \gamma$ become bigger the droop of the height of wave crest is faster. 


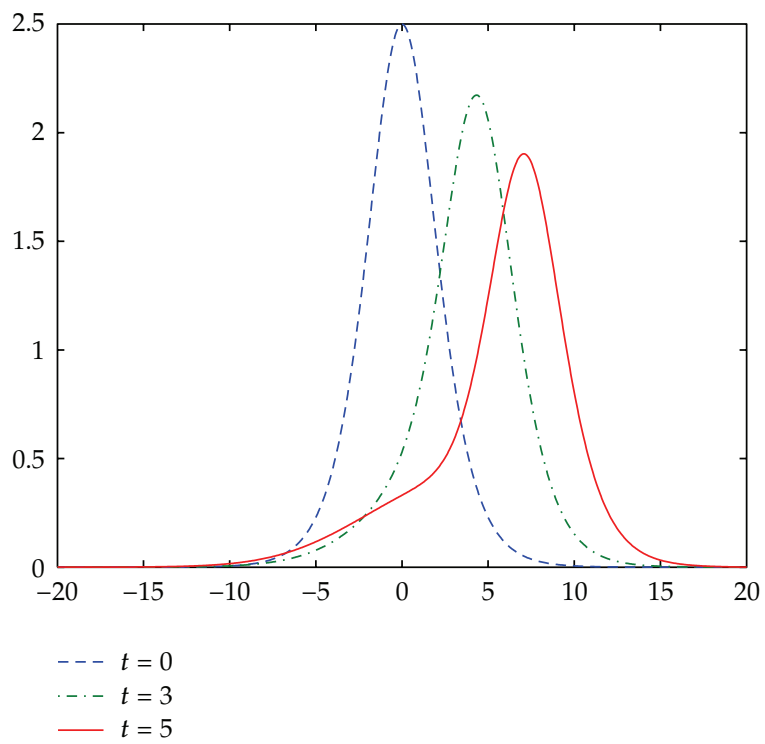

Figure 1: When $v=\gamma=0.2$, the wave graph of $u$ at various time.

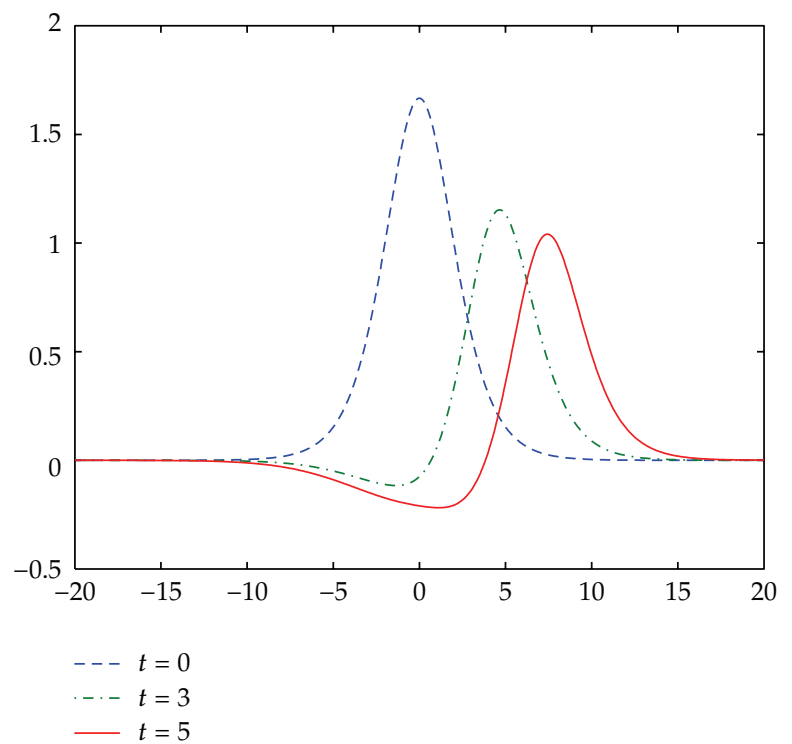

Figure 2: When $v=\gamma=0.2$, the wave graph of $\rho$ at various time.

\section{Acknowledgments}

This work was supported by the Sichuan province application of technology research and development project (no. 2010JY0058), the youth research foundation of Sichuan University (no. 2009SCU11113) and the research fund of key disciplinary of application mathematics of Xihua University (Grant no. XZD0910-09-1). 


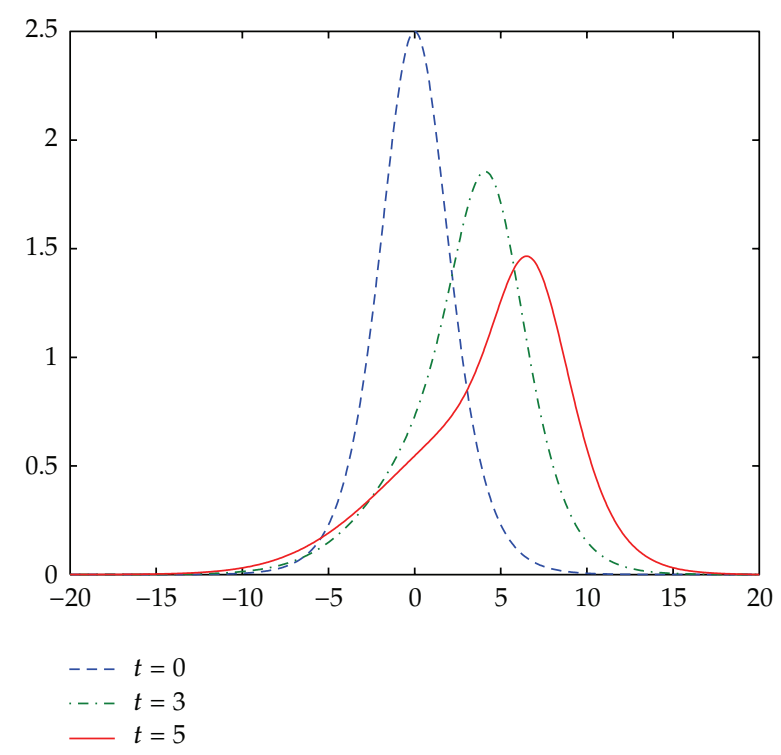

Figure 3: When $v=r=0.5$, the wave graph of $u$ at various time.

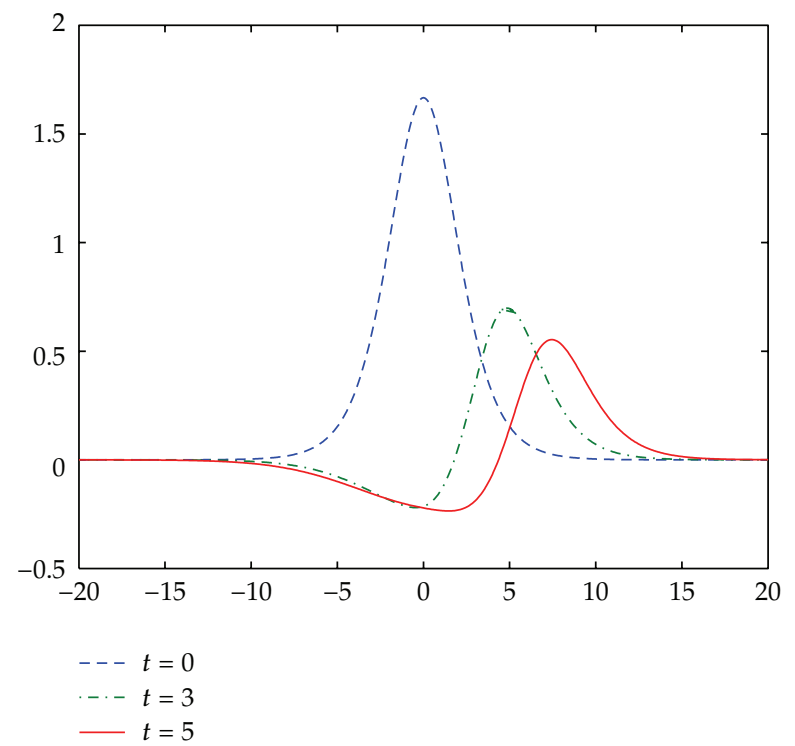

Figure 4: When $v=\gamma=0.5$, the wave graph of $\rho$ at various time.

\section{References}

[1] C. E. Seyler and D. L. Fenstermacher, "A symmetric regularized-long-wave equation," Physics of Fluids, vol. 27, no. 1, pp. 4-7, 1984.

[2] J. Albert, "On the decay of solutions of the generalized Benjamin-Bona-Mahony equations," Journal of Mathematical Analysis and Applications, vol. 141, no. 2, pp. 527-537, 1989.

[3] C. J. Amick, J. L. Bona, and M. E. Schonbek, "Decay of solutions of some nonlinear wave equations, "Journal of Differential Equations, vol. 81, no. 1, pp. 1-49, 1989. 
[4] T. Ogino and S. Takeda, "Computer simulation and analysis for the spherical and cylindrical ionacoustic solitons," Journal of the Physical Society of Japan, vol. 41, no. 1, pp. 257-264, 1976.

[5] V. G. Makhankov, "Dynamics of classical solitons (in nonintegrable systems)," Physics Reports C, vol. 35, no. 1, pp. 1-128, 1978.

[6] P. A. Clarkson, "New similarity reductions and Painlevé analysis for the symmetric regularised long wave and modified Benjamin-Bona-Mahoney equations," Journal of Physics A, vol. 22, no. 18, pp. 38213848, 1989.

[7] I. L. Bogolubsky, "Some examples of inelastic soliton interaction," Computer Physics Communications, vol. 13, no. 3, pp. 149-155, 1977.

[8] B. L. Guo, "The spectral method for symmetric regularized wave equations," Journal of Computational Mathematics, vol. 5, no. 4, pp. 297-306, 1987.

[9] J. D. Zheng, R. F. Zhang, and B. Y. Guo, "The Fourier pseudo-spectral method for the SRLW equation," Applied Mathematics and Mechanics, vol. 10, no. 9, pp. 801-810, 1989.

[10] J. D. Zheng, "Pseudospectral collocation methods for the generalized SRLW equations," Mathematica Numerica Sinica, vol. 11, no. 1, pp. 64-72, 1989.

[11] Y. D. Shang and B. L. Guo, "Legendre and Chebyshev pseudospectral methods for the generalized symmetric regularized long wave equations," Acta Mathematicae Applicatae Sinica, vol. 26, no. 4, pp. 590-604, 2005.

[12] Y. Bai and L. M. Zhang, "A conservative finite difference scheme for symmetric regularized long wave equations," Acta Mathematicae Applicatae Sinica, vol. 30, no. 2, pp. 248-255, 2007.

[13] T. Wang, L. Zhang, and F. Chen, "Conservative schemes for the symmetric regularized long wave equations," Applied Mathematics and Computation, vol. 190, no. 2, pp. 1063-1080, 2007.

[14] T. C. Wang and L. M. Zhang, "Pseudo-compact conservative finite difference approximate solution for the symmetric regularized long wave equation," Acta Mathematica Scientia A, vol. 26, no. 7, pp. 1039-1046, 2006.

[15] T. C. Wang, L. M. Zhang, and F. Q. Chen, "Pseudo-compact conservative finite difference approximate solutions for symmetric regularized-long-wave equations," Chinese Journal of Engineering Mathematics, vol. 25, no. 1, pp. 169-172, 2008.

[16] Y. Shang, B. Guo, and S. Fang, "Long time behavior of the dissipative generalized symmetric regularized long wave equations," Journal of Partial Differential Equations, vol. 15, no. 1, pp. 35-45, 2002.

[17] Y. D. Shang and B. L. Guo, "Global attractors for a periodic initial value problem for dissipative generalized symmetric regularized long wave equations," Acta Mathematica Scientia A, vol. 23, no. 6, pp. 745-757, 2003.

[18] B.-1. Guo and Y.-D. Shang, "Approximate inertial manifolds to the generalized symmetric regularized long wave equations with damping term," Acta Mathematicae Applicatae Sinica, vol. 19, no. 2, pp. 191204, 2003.

[19] Y. D. Shang and B. L. Guo, "Exponential attractor for the generalized symmetric regularized long wave equation with damping term," Applied Mathematics and Mechanics, vol. 26, no. 3, pp. 259-266, 2005.

[20] F. Shaomei, G. Boling, and Q. Hua, "The existence of global attractors for a system of multidimensional symmetric regularized wave equations," Communications in Nonlinear Science and Numerical Simulation, vol. 14, no. 1, pp. 61-68, 2009.

[21] J. Hu, Y. Xu, and B. Hu, "A linear difference scheme for dissipative symmetric regularized long wave equations with damping term," Boundary Value Problems, vol. 2010, Article ID 781750, 16 pages, 2010.

[22] S. K. Chung, "Finite difference approximate solutions for the Rosenau equation," Applicable Analysis, vol. 69 , no. $1-2$, pp. 149-156, 1998.

[23] B. Hu, Y. Xu, and J. Hu, "Crank-Nicolson finite difference scheme for the Rosenau-Burgers equation," Applied Mathematics and Computation, vol. 204, no. 1, pp. 311-316, 2008.

[24] F. E. Browder, "Existence and uniqueness theorems for solutions of nonlinear boundary value problems," Proceedings of Symposia in Applied Mathematics, vol. 17, pp. 24-49, 1965.

[25] T. Wang and B. Guo, "A robust semi-explicit difference scheme for the Kuramoto-Tsuzuki equation," Journal of Computational and Applied Mathematics, vol. 233, no. 4, pp. 878-888, 2009. 


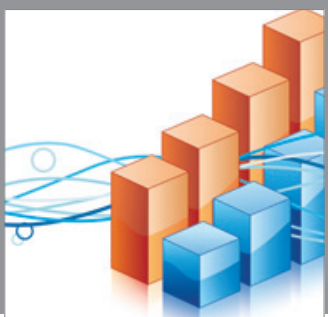

Advances in

Operations Research

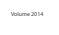

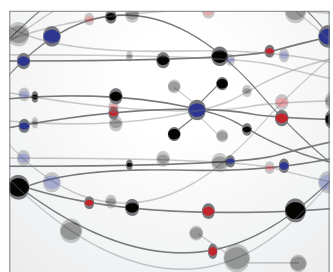

\section{The Scientific} World Journal
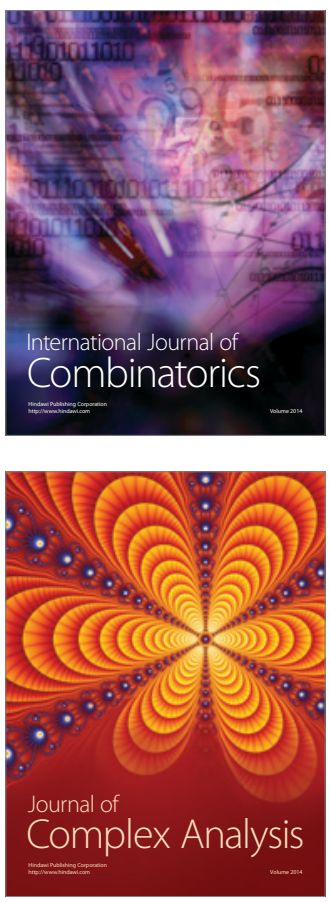

International Journal of

Mathematics and

Mathematical

Sciences
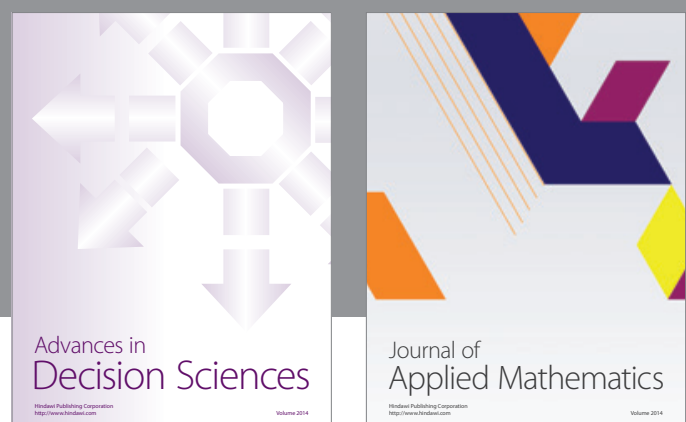

Journal of

Applied Mathematics
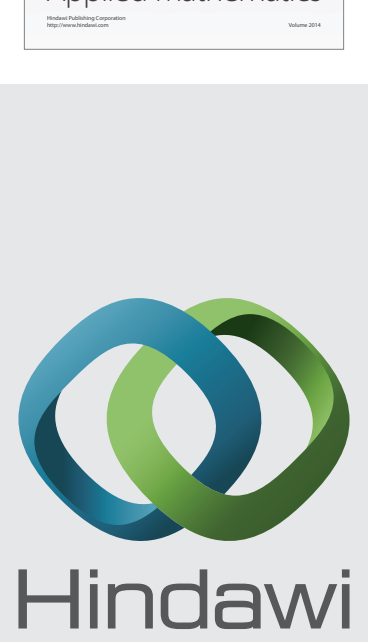

Submit your manuscripts at http://www.hindawi.com
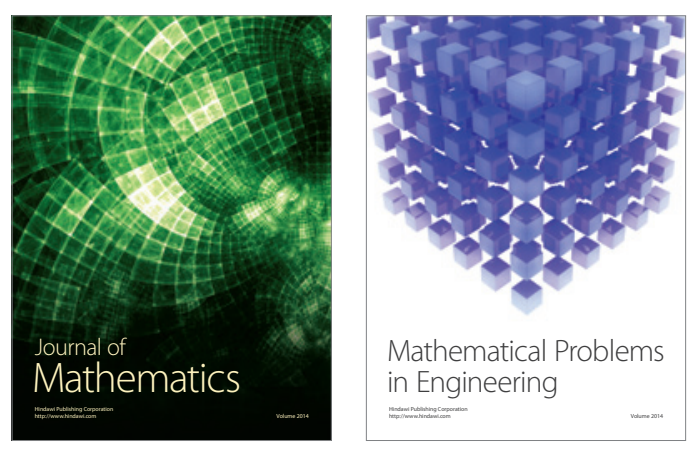

Mathematical Problems in Engineering
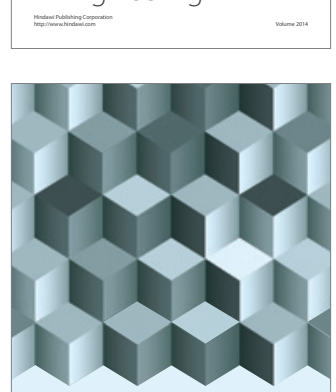

Journal of

Function Spaces
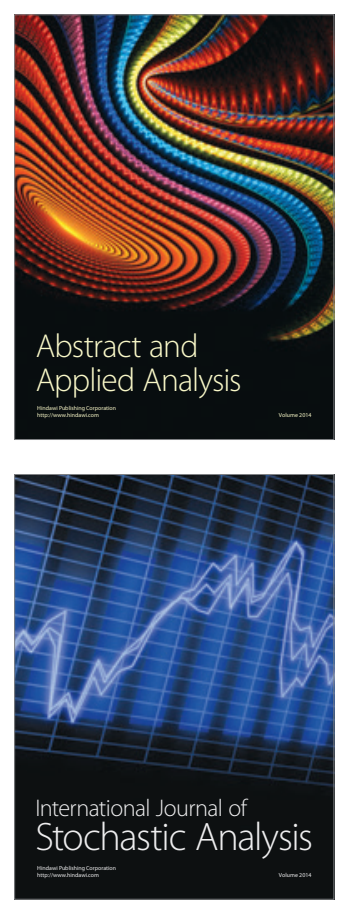

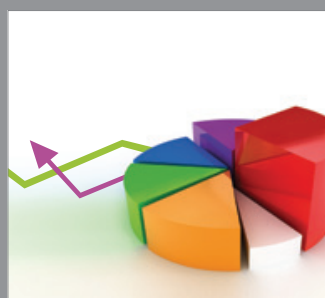

ournal of

Probability and Statistics

Promensencen
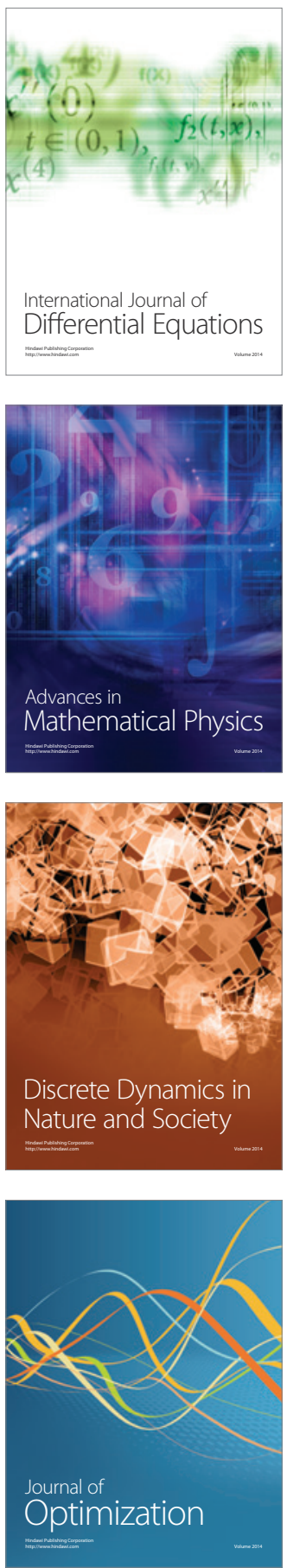